\title{
Study on the multiple diffraction for UWB signals under NLOS environment in WSNs
}

\author{
HAN Tao ${ }^{a}$, KUANG Zhaoxuan ${ }^{b}$, WANG Hongcheng ${ }^{c}$, LI Xiuping ${ }^{d}$ \\ College of Electrical Engineering, Dongguan University of Technology, DongguanGuangdong \\ 523808, China \\ ahant@dgut.edu.cn, bkuangzhx@dgut.edu.cn, 'wanghc@dgut.edu.cn, 'lixp@dgut.edu.cn
}

Keywords: ultra-wideband (UWB); uniform theory of diffraction (UTD); multiple diffraction; pulse distortion

\begin{abstract}
A time domain (TD) hybrid solution based on uniform theory of diffraction-physical optics (UTD-PO) is presented to analyze the multiple diffraction under nonline-of-sight (NLOS) environment where an obstacle causes shadowing of the transmitter from the receiver in wireless sensor networks (WSNs). The channel impulse response of the model is derived, which utilizes a recursion relation and does not need to incorporate the slope diffraction in the transition zone. The received signals can be predicted through a simple operation of convolution, which provides a faster and easier final solution for UWB radio propagation and significantly reduces the computational time. The TD result can be applied to analyze the UWB pulse distortion by multiple diffraction under NLOS environment in WSNs.
\end{abstract}

\section{Introduction}

Emerging of application of ultra-wide band (UWB) are foreseen for WSNs that combine low to medium rate communications over distances of $100 \mathrm{~m}[1]$. When sensors are placed in different areas, a nonline-of-sight propagation is encountered very often, sometimes in military communications[2]. During propagation in urban areas, UWB signals usually undergo double or multiple diffraction before they are received. The recent interest in the use of UWB technique has motivated the propagation analysis of UWB channel characteristics due to the fact that the propagation characteristics play a fundamental role in the design and implementation of the UWB systems[3].

In the case of the UWB propagation, using the impulse response of a scattering object in the TD is more convenient than applying numerical inverse Fourier transform algorithms to convert frequency domain (FD) solutions into the TD, since the bandwidth of UWB signals is so large that the distortion of an UWB pulse is frequency dependent. Karousos and Tzaras[4] have presented a TD-UTD solution which successfully analyzes multiple diffraction through the incorporation of the slope diffraction. Nevertheless, it is difficult to apply their solution in multiple diffraction where the number of obstacles is large since their solution has to incorporate the slope diffraction[5] in the transition zone, which increases both the mathematical complexity and the computational efficiency of the solution. Han and Long[6] have presented a hybrid UTD-PO solution to derive the impulse response of Bertoni's urban propagation configuration where the transmitting antenna is located at a certain distance from the array of buildings with rectangular cross sections.

In this paper, the work[6] is extended to another model, in which an array of knife edges are surrounded by an obstacle modeled as a knife edge, considering spherical-wave incidence. The impulse response of the channel is obtained and it is convolved with the transmitted pulse to produce the received signal for analysis the TD characteristics for the abovementioned model. Based on UTD-PO for multiple diffraction, the received signal may be seen as the average of possible signals from different knife edges, which avoids the incorporation of the slope diffraction. The predicted signal is compared with the numerical inverse fast Fourier transforms of the FD solution. The results show a very good agreement between the two solutions with our solution offering much shorter computation times due to a recursive relation in which only single diffractions are involved in the calculations. 


\section{Propagation environment}

A scheme of the propagation environment considered is shown in Figure 1, the $n$ parallel knife edges with the same height $h_{k e}$ are adjacent to each other with the same distance $w$.The obstacle of height $h_{w}$ is placed at a certain distance $d_{2}$ from the aforementioned knife edges. The transmitter with height $h_{t x}$ is located a distance $d_{1}$ from the obstacle. The receiver, with the same height as the knife edges, is located at a distance $w$ from the array of knife edges. In addition, the multiple diffraction occurs for a given position of the transmitter, receiver and knife edges within the shadow of the obstacle.

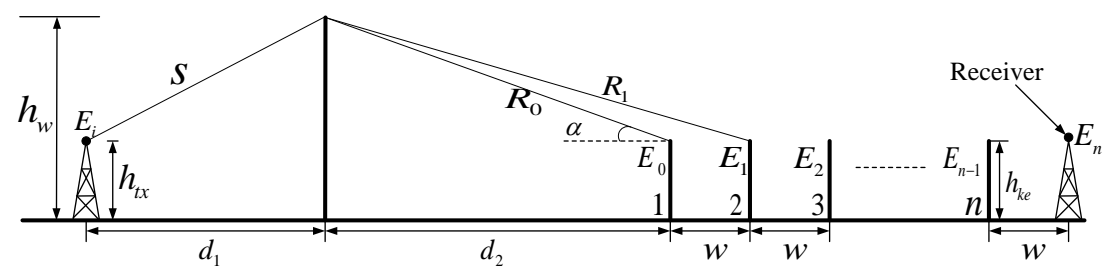

Fig. 1 A scheme of the propagation environment considered for UWB signals under NLOS environment

\section{Frequency domain model}

According to the solution in [6], the received signal in figure 1 can be seen as the average of possible signals from different knife edges. The electrical field in the FD at the receiver indicated in Figure 1 for $n \geq 1$ can be expressed as

$$
\begin{aligned}
E_{n}(\omega)= & \frac{1}{n}\left\{E_{0}(\omega)\left[\frac{E_{0 n}(\omega)}{E_{00}(\omega)} \exp \left(-j k\left(R_{n}-R_{0}\right)\right)+\sqrt{\frac{R_{0}}{n w\left(R_{0}+n w\right)}} \cdot D_{k e}(\omega, n) \cdot \exp (-j k n w)\right]\right. \\
& +\sum_{m=1}^{n-1} E_{m}(\omega)\left[\frac{R_{0}}{R_{n-m}} \exp \left(-j k\left(R_{n}-R_{m}\right)\right)\right. \\
& \left.\left.+\sqrt{\frac{R_{0}}{(n-m) w\left[R_{0}+(n-m) w\right]}} \cdot D_{k e}(\omega, n-m) \cdot \exp (-j k(n-m) w)\right]\right\}
\end{aligned}
$$

where

$$
\begin{aligned}
E_{0}(\omega) & =\frac{E_{i}}{s} \exp (-j k s) \cdot \sqrt{\frac{s}{R_{0}\left(R_{0}+s\right)}} \cdot D_{w}(\omega, n=0) \cdot \exp \left(-j k R_{0}\right) \\
& =E_{00}(\omega) \cdot \exp \left(-j k R_{0}\right)
\end{aligned}
$$

is the field intensity arriving at the first knife edge, $k$ is the wave number, $E_{i}$ is the relative amplitude of a spherical source,

$$
\begin{gathered}
E_{0 n}(\omega)=\frac{E_{i}}{s} \exp (-j k s) \cdot \sqrt{\frac{s}{R_{n}\left(R_{n}+s\right)}} \cdot D_{w}(\omega, n) \\
R_{n}=\sqrt{\left(h_{w}-h_{k e}\right)^{2}+\left(d_{2}+n w\right)^{2}} \\
D_{k e}(\omega, n)=D_{k e}^{s, h}\left(\phi=\frac{3 \pi}{2}, \phi^{\prime}=\frac{\pi}{2}+\alpha, L=\frac{R_{0} n w}{R_{0}+n w}\right) \\
D_{w}(\omega, n)=D_{w}^{s, h}\left(\phi=2 \pi-\arctan \left(\frac{d_{2}+n w}{h_{w}-h_{k e}}\right), \phi^{\prime}=\arcsin \left(\frac{d_{1}}{s}\right), L=\frac{s R_{n}}{s+R_{n}}\right)
\end{gathered}
$$

$D_{w}^{s, h}\left(\phi, \phi^{\prime}, L\right)$ and $D_{k e}^{s, h}\left(\phi, \phi^{\prime}, L\right)$ are the diffraction coefficients given in [7] for a perfectly conducting obstacle and a perfectly conducting knife edge, respectively. 


\section{Time domain model}

The recent advances in the development of UWB communications systems have given rise to an increasing interest in analytically deriving the TD impulse response for some relatively environments. In this section, the impulse response of the abovementioned environment will be derived with the help of the Laplace transform. Therefore, (1) is translated into the TD as:

$$
\begin{aligned}
e_{n}(t)= & \frac{1}{n}\left\{e_{0 n}(t) * \delta\left(t-\frac{R_{n}}{c}\right)+\sqrt{\frac{R_{0}}{n w\left(R_{0}+n w\right)}} e_{0}(t) * d_{k e}(t, n)\right. \\
& +\sum_{m=1}^{n-1} e_{m}(t) *\left[\frac{R_{0}}{R_{n-m}} \cdot \delta\left(t-\frac{R_{n}-R_{m}}{c}\right)+\sqrt{\frac{R_{0}}{(n-m) w+\left[R_{0}+(n-m) w\right]}}\right. \\
& \left.\left.\cdot d_{k e}(t, n-m) * \delta\left(t-\frac{(n-m) w}{c}\right)\right]\right\}
\end{aligned}
$$

where

$$
e_{0 n}(t)=\frac{E_{i}}{s} \cdot \sqrt{\frac{s}{R_{n}\left(R_{n}+s\right)}} \cdot \delta\left(t-\frac{s}{c}\right) * d_{w}(t, n)
$$

$c$ is the speed of light, symbol ' $*$ ', denotes the convolution operation, $\delta(\cdot)$ is the Diracdetla function, $d_{k e}(t, n)$ and $d_{w}(t, n)$ are the TD-UTD diffraction coefficients for a perfectly conducting knife edge and a perfectly conducting obstacle, respectively.

For the case of a perfectly conducting knife edge, the TD diffraction coefficient is given by [8]

$$
\begin{gathered}
d^{s, h}(t)=\frac{-1}{2 \sqrt{2 \pi}}\left[\frac{f\left(X^{-}, t\right)}{\cos \left[\left(\phi-\phi^{\prime}\right) / 2\right]} \mp \frac{f\left(X^{+}, t\right)}{\cos \left[\left(\phi+\phi^{\prime}\right) / 2\right]}\right] \\
f\left(X^{ \pm}, t\right)=\frac{X^{ \pm}}{\sqrt{\pi c t}\left(t+X^{ \pm} / c\right)}
\end{gathered}
$$

with $X^{ \pm}=2 L \cos ^{2}\left[\left(\phi \pm \phi^{\prime}\right) / 2\right], L$ is the distance parameter in (5) and (6).

\section{Results}

Throughout this Letter, the excitation pulse used in the simulations is the second-order derivative of the Gaussian pulse which can be expressed as

$$
s=\frac{1}{\tau} \sqrt{\frac{\tau}{3 \sqrt{\pi / 2}}} \cdot\left(1-2 \frac{t^{2}}{\tau^{2}}\right) \cdot \exp \left(-\frac{t^{2}}{\tau^{2}}\right)
$$

where $\tau$ is the parameter used to control the width of the pulse with a value of $\tau=0.1 \mathrm{~ns}$. The environment parameters in Figure 1 are as follows: $h_{t x}=2 \mathrm{~m}, h_{k e}=3 \mathrm{~m}, h_{w}=3.5 \mathrm{~m}, d_{1}=5 \mathrm{~m}$, $d_{2}=10 \mathrm{~m}, w=2 \mathrm{~m}, n=5$ and $\gamma=\pi / 2$. The value of $h_{w}$ and the number of $n$ can vary according to our need.

In order to validate the proposed formulas, a comparison with the numerical inverse fast Fourier transforms of the FD solution can be observed in Figure 2. It is assumed that the transmitter sends a soft polarized pulse, and then a hard polarized pulse and the interval of the two pulses is $10 \mathrm{~ns}$. We neglect a time delay factor common to two pulses. As can be seen in Figure 2, the received signals for both soft and hard polarizations are attenuated and distorted greatly, since the transmitter and the receiver are in the shadow region of the obstacle. In addition, there is a good agreement between the results of our solution and those with the numerical inverse fast Fourier transforms of the FD solution. Furthermore, it should be noticed that the two evaluated polarizations can not give significantly different results in the shape of the received UWB signals for the abovementioned environment, which also occurs in the environment[6] where no obstacle is included. 


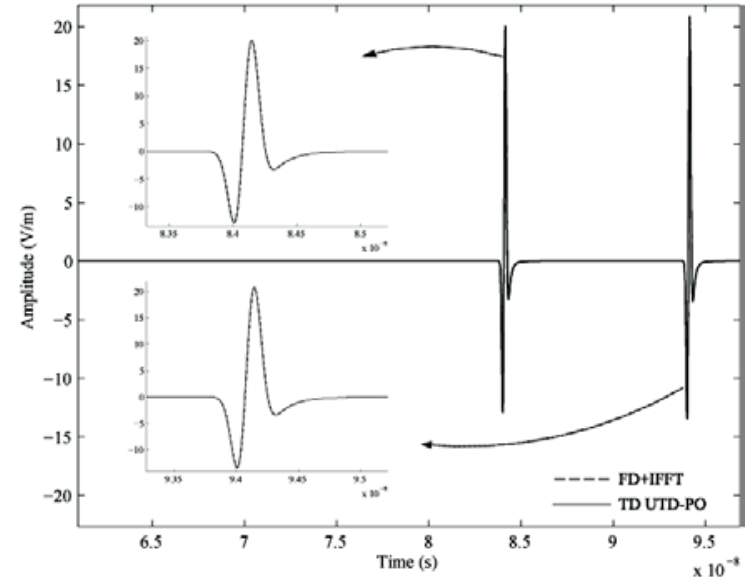

Fig. 2 Received signals in the gazing incidence of five knife edges

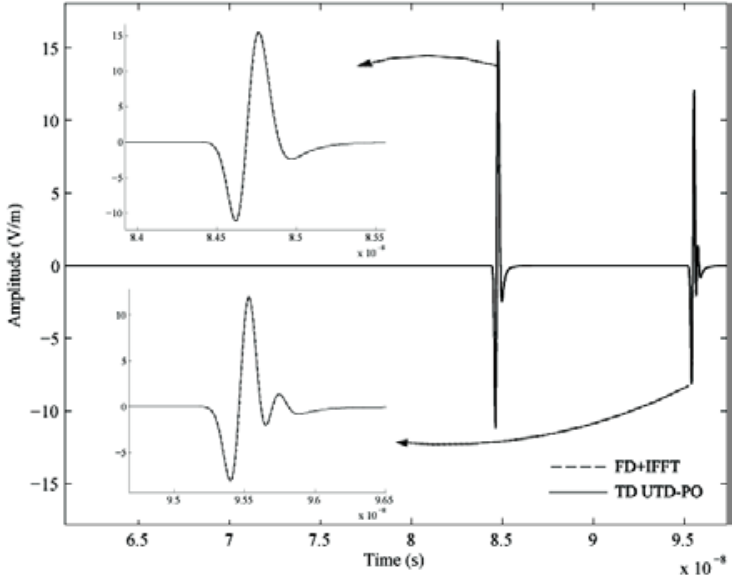

Fig. 3 Received signals for two different obstacle height $h_{w}$

In Figure 3, the received signals for two different obstacle height $h_{w}$ can be observed. It is assumed that the transmitter sends a hard polarized pulse every $10 \mathrm{~ns}$. In each transmission time, the obstacle height $h_{w}$ is $4 \mathrm{~m}$ and $4.5 \mathrm{~m}$, respectively. It should be noted that when the height of the obstacle grows, the knife edges and receiver are getting deeper into the shadow region of the obstacle and the incident angle on the knife edges becomes lager. As a result, the diffraction losses caused by the obstacle increase and the losses produced by the knife edges are reduced. As can be seen in Figure 3 , the second received signal is more attenuated and distorted than the first one since the growth in the losses produced by the obstacle exceeds the decrease in the losses caused by the knife edges when the knife edges and the receiver are getting deeper into the shadow region of the obstacle. In addition, the inter-symbol interference appears when the height of the obstacle $h_{w}$ increases since the pulses received at the antenna are from different paths. Also, the proposed TD solution agrees very well with the numerical inverse fast Fourier transforms of the FD solution.

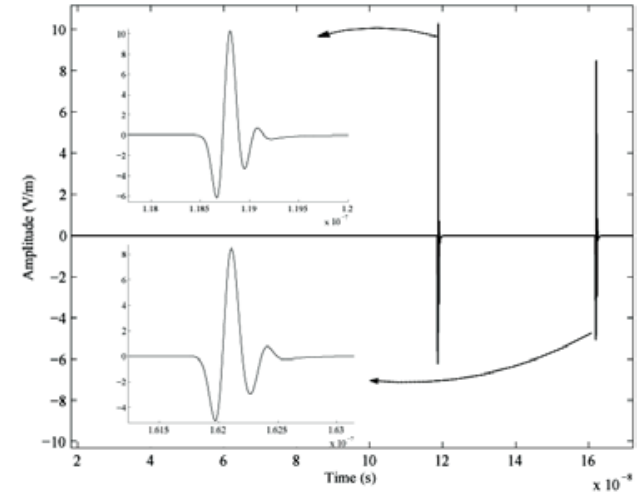

Fig. 4 Received signals for two different number of knife edges

Finally, the received hard polarized signals through the proposed solution as a function of the number of knife edges $n$ are shown in Figure 4. It is assumed that the height of the obstacle $h_{w}$ is 4.5 $\mathrm{m}$ and the transmitter sends pulses every $10 \mathrm{~ns}$. In each transmission time, the number of knife edges $n$ is 10 and 15, respectively. It should be noted that the solution given in [4] has to incorporate $2^{n}$ rays in the case of $n$ knife edges, so it can hardly be applied in multiple diffraction when the number of knife edges is large. In addition, the proposed solution with less mathematical complexity significantly shortens the time of computation due to the absence of the slope diffraction terms, when the number of knife edges is increasing. This can be seen in Table 1, where the average ratios of the computation times of the two methods $T_{T D[4]} / T_{T D[U T D-P O]}$ are presented with 4, 5, 6 knife edges. It can 
be noticed that the proposed solution has better computational efficiency, when the number of knife edges is increased.

Table 1 The ratios of computation times of the two methods

\begin{tabular}{c|c}
\hline Number of buildings & $T_{T D[4]} / T_{T D[U T D-P O]}$ \\
\hline 3 & 1.93 \\
4 & 2.59 \\
5 & 3.87 \\
\hline
\end{tabular}

\section{Conclusions}

This paper derives the impulse response based on UTD-PO in the TD for the propagation environment where an obstacle obstructs the propagation path between the transmitter and the knife edges. The shape of the received UWB signals can be determined through an operation of convolution, which has been validated through the comparison with the numerical inverse fast Fourier transforms of the FD solution. The solution has a good computational efficiency due to the recursive technique that utilizes. The TD result can be used to analyze the characteristics of the UWB signals through the propagation environment where multiple diffraction under NLOS environment is considered in WSNs.

\section{Acknowledgements}

This work was supported jointly by the National Natural Science Foundation of China (Grant No. 61308019), the Natural Science Foundation of Guangdong (Grant No. S2013010013261) and the Research Project of the Economic \& Information Commission of Guangdong Province.

\section{References}

[1] Oner M. On the Spectral Correlation of UWB Impulse Radio Signals [J]. IEEE Commun Lett., 2008, 12(10): 714-716

[2] Gezici S, Tian Z, Giannakis G B, Kobayahsi H, et al. Location via ultra-wideband radios [J]. IEEE Signal Process. Mag., 2005, 22(4): 70-84

[3] Zhou C, Qiu R. Pulse distortion caused by cylinder diffraction and its impact on UWB communications [J]. IEEE Transactions on Vehicular Technology, 2007, 56(4): 2385-2391

[4] Karousos A, Tzaras C. Multiple time-domain diffraction for UWB signals [J]. IEEE Transactions on Antennas and Propagation, 2008, 56(5): 1420-1427

[5] Andersen J B. UTD multiple-edge transition zone diffraction [J]. IEEE Transactions on Antennas and Propagation, 1997, 45(7): 1093-1097

[6] Han T, Long Y. Time-domain UTD-PO analysis of a UWB pulse distortion by multiple-building diffraction [J]. IEEE Antennas and Wireless Propagation Letters, 2010, 9: 795-798

[7] Mcnamara D A, Pistorius C W I, Malherbe J A G. Introduction to the uniform geometrical theory of diffraction [M]. Norwood: Artech House, 1990: 191-197

[8] P. Rousseau and P. Pathak. Time-Domain Uniform Geometrical Theory of Diffraction for a Curved Wedge [J]. IEEE Transactions on Antennas and Propagation, 1995, 43(12): 1375-1382 